\title{
Molecular gas in AzTEC/C159: a star-forming disk galaxy 1.3 Gyr after the Big Bang
}

\author{
E. F. Jiménez-Andrade ${ }^{1,2}$, B. Magnelli ${ }^{1}$, A. Karim ${ }^{1}$, G. C. Jones ${ }^{3,4}$, C. L. Carilli4 ${ }^{4,5}$, E. Romano-Díaz ${ }^{1}$,
} C. Gómez-Guijarro ${ }^{6}$, S. Toft ${ }^{6}$, F. Bertoldi ${ }^{1}$, D. A. Riechers ${ }^{7}$, E. Schinnerer ${ }^{8}$, M. Sargent ${ }^{9}$, M. J. Michałowski ${ }^{10}$, F. Fraternali ${ }^{11,12}$, J. G. Staguhn ${ }^{13,14}$, V. Smolčić ${ }^{15}$, M. Aravena ${ }^{16}$, K. C. Harrington ${ }^{1,2}$, K. Sheth ${ }^{17}$, P. L. Capak ${ }^{18,19}$, A. M. Koekemoer ${ }^{20}$, E. van Kampen ${ }^{21}$, M. Swinbank ${ }^{22}$, A. Zirm ${ }^{6,23}$, G. E. Magdis ${ }^{6,24}$, and F. Navarrete ${ }^{25}$

${ }^{1}$ Argelander Institute for Astronomy, University of Bonn, Auf dem Hügel 71, 53121 Bonn, Germany e-mail: ericja@astro.uni-bonn.de

${ }^{2}$ International Max Planck Research School of Astronomy and Astrophysics, Universities of Bonn and Cologne, Bonn, Germany

${ }^{3}$ Physics Department, New Mexico Institute of Mining and Technology, 801 Leroy Pl, Socorro, NM 87801, USA

${ }^{4}$ National Radio Astronomy Observatory, 1003 Lopezville Road, Socorro, NM 87801, USA

${ }^{5}$ Cavendish Astrophysics Group, University of Cambridge, Cambridge CB3 OHE, UK

${ }^{6}$ Dark Cosmology Centre, Niels Bohr Institute, University of Copenhagen, Juliane Maries Vej 30, 2100 Copenhagen, Denmark

${ }^{7}$ Department of Astronomy, Cornell University, Space Sciences Building, Ithaca, NY 14853, USA

${ }^{8}$ Max Planck Institute for Astronomy, Königstuhl 17, 69117 Heidelberg, Germany

9 Astronomy Centre, Department of Physics and Astronomy, University of Sussex, Brighton BN1 9QH, UK

${ }_{10}^{10}$ Astronomical Observatory Institute, Faculty of Physics, Adam Mickiewicz University, ul. Słoneczna 36, 60-286 Poznań, Poland

${ }^{11}$ Department of Physics and Astronomy, University of Bologna, viale Berti Pichat 6/2, 40127 Bologna, Italy

${ }^{12}$ Kapteyn Astronomical Institute, Postbus 800, 9700 AV Groningen, The Netherlands

${ }^{13}$ NASA Goddard Space Flight Center, Code 665, Greenbelt, MD 20771, USA

${ }^{14}$ Department of Physics and Astronomy, Johns Hopkins University, Baltimore, MD 21218, USA

15 Department of Physics, Faculty of Science, University of Zagreb, Bijenička cesta 32, 10000 Zagreb, Croatia

${ }^{16}$ Núcleo de Astronomía, Facultad de Ingeniería y Ciencias, Universidad Diego Portales, Av. Ejército 441, Santiago, Chile

${ }^{17}$ Science Mission Directorate, NASA Headquarters, Washington, DC 20546-0001, USA

${ }^{18}$ Infrared Processing and Analysis Center, California Institute of Technology, MC 100-22, 770 South Wilson Ave., Pasadena, CA 91125, USA

${ }^{19}$ Spitzer Science Center, California Institute of Technology, Pasadena, CA 91125, USA

20 Space Telescope Science Institute, 3700 San Martin Drive, Baltimore, MD 21218, USA

${ }^{21}$ European Southern Observatory, Karl-Schwarzschild-Strasse 2, 85748 Garching bei Muenchen, Germany

22 Centre for Extragalactic Astronomy, Durham University, South Road, Durham DH1 3LE, UK

${ }^{23}$ Greenhouse Software, 3rd Floor, 110 5th Avenue, New York, NY 10011, USA

${ }^{24}$ Institute for Astronomy, Astrophysics, Space Applications and Remote Sensing, National Observatory of Athens, 15236 Athens, Greece

25 Max Planck Institute for Radioastronomy, Auf dem Hügel 69, 53121 Bonn, Germany

Received 27 October 2017 / Accepted 9 February 2018

\section{ABSTRACT}

We studied the molecular gas properties of AzTEC/C159, a star-forming disk galaxy at $z=4.567$, in order to better constrain the nature of the high-redshift end of the submillimeter-selected galaxy (SMG) population. We secured ${ }^{12} \mathrm{CO}$ molecular line detections for the $J=2 \rightarrow 1$ and $J=5 \rightarrow 4$ transitions using the Karl G. Jansky Very Large Array (VLA) and the NOrthern Extended Millimeter Array (NOEMA) interferometer. The broad $\left(F W H M \sim 750 \mathrm{~km} \mathrm{~s}^{-1}\right)$ and tentative double-peaked profiles of the two ${ }^{12} \mathrm{CO}$ lines are consistent with an extended molecular gas reservoir, which is distributed in a rotating disk, as previously revealed from [CII] $158 \mu \mathrm{m}$ line observations. Based on the ${ }^{12} \mathrm{CO}(2 \rightarrow 1)$ emission line, we derived $L_{\mathrm{CO}}^{\prime}=(3.4 \pm 0.6) \times 10^{10} \mathrm{~K} \mathrm{~km} \mathrm{~s}^{-1} \mathrm{pc}^{2}$, which yields a molecular gas mass of $M_{\mathrm{H}_{2}}\left(\alpha_{\mathrm{CO}} / 4.3\right)=(1.5 \pm 0.3) \times 10^{11} M_{\odot}$ and unveils a gas-rich system with $\mu_{\mathrm{gas}}\left(\alpha_{\mathrm{CO}} / 4.3\right) \equiv M_{\mathrm{H}_{2}} / M_{\star}=3.3 \pm 0.7$. The extreme star formation efficiency of AzTEC/C159, parametrized by the ratio $L_{\mathrm{IR}} / L_{\mathrm{CO}}^{\prime}=(216 \pm 80) L_{\odot}\left(\mathrm{K} \mathrm{km} \mathrm{s}^{-1} \mathrm{pc}^{2}\right)^{-1}$, is comparable to merger-driven starbursts such as local ultra-luminous infrared galaxies and SMGs. Likewise, the ${ }^{12} \mathrm{CO}(5 \rightarrow 4) / \mathrm{CO}(2 \rightarrow 1)$ line brightness temperature ratio of $r_{52}=0.55 \pm 0.15$ is consistent with high-excitation conditions as observed in SMGs. Based on mass budget considerations, we constrained the value for the $L_{\mathrm{CO}}^{\prime}-\mathrm{H}_{2}$ mass conversion factor in AzTEC/C159, that is, $\alpha_{\mathrm{CO}}=3.9_{-1.3}^{+2.7} M_{\odot} \mathrm{K}^{-1} \mathrm{~km}^{-1} \mathrm{spc}^{-2}$, which is consistent with a self-gravitating molecular gas distribution as observed in local star-forming disk galaxies. Cold gas streams from cosmological filaments might be fueling a gravitationally unstable gas-rich disk in AzTEC/C159, which breaks into giant clumps and forms stars as efficiently as in merger-driven systems and generates high gas excitation. These results support the evolutionary connection between AzTEC/C159-like systems and massive quiescent disk galaxies at $z \sim 2$.

Key words. galaxies: high-redshift - galaxies: formation - galaxies: ISM - ISM: molecules 


\section{Introduction}

Submillimeter-selected galaxies (SMGs), which are gas-rich starbursts at high redshifts (e.g., Blain et al. 2002; Tacconi et al. 2006, 2008; Narayanan et al. 2010; Casey et al. 2014), might be the progenitors of compact quiescent galaxies $\left(\log \left(M_{\star} / M_{\odot}\right)>11\right)$ at $z \sim 2$ (e.g., Simpson et al. 2014; Toft et al. 2014; Ikarashi et al. 2015; Oteo et al. 2016, 2017). It is believed that the intense starburst episode can be followed by an active galactic nucleus (AGN) phase that eventually quenches the star formation (Hopkins et al. 2006; Wuyts et al. 2010; Hickox et al. 2012; Steinhardt \& Speagle 2014). However, there is a lack of consensus on the physical mechanisms driving the extreme production of stars in SMGs. It has been proposed that compact starbursts might be fueled via major gas-rich mergers (e.g., Walter et al. 2009; Narayanan et al. 2010; Hayward et al. 2011, 2012; Alaghband-Zadeh et al. 2012; Riechers et al. 2013, 2014). An alternative scenario involves the smooth infall and accretion of cold gas from the intergalactic medium that could also drive intense star formation in massive highly unstable high-redshift galaxies (e.g., Dekel et al. 2009a; Kereš et al. 2009a,b; Davé et al. 2010; Hodge et al. 2012; Romano-Díaz et al. 2014; Feng et al. 2015; Anglés-Alcázar et al. 2017).

To constrain the origin of SMGs and their possible evolutionary path, much effort has been spent in building statistically complete and unbiased samples of these objects (e.g., Banerji et al. 2011; Yun et al. 2012; Hodge et al. 2013; Strandet et al. 2016; Brisbin et al. 2017; Michałowski et al. 2017). Particular emphasis has been placed on the recently discovered high-redshift tail $(4<z<6)$ of the SMG population (e.g., Daddi et al. 2009a,b; Capak et al. 2008, 2011; Coppin et al. 2009; Knudsen et al. 2010; Smolčić et al. 2011; Barger et al. 2012; Walter et al. 2012; Ivison et al. 2016). By exploring the physical properties of these $z>4$ systems, one might further strengthen the evolutionary link between high-redshift SMGs and massive quiescent galaxies at $z \sim 2$ (e.g., Fudamoto et al. 2017), and provide constraints on cosmological models that aim to reproduce the extreme and massive environments of early SMGs (e.g., Baugh et al. 2005; Dwek et al. 2011; Hayward et al. 2011, 2013; Ferrara et al. 2016). However, probing the nature of these systems is observationally expensive (e.g., Hodge et al. 2012) and somewhat complex. Although gravitationally lensed sources might overcome the issue of timeconsuming follow-up observations (e.g., Strandet et al. 2016; Harrington et al. 2016, 2018), their derived quantities might be affected by lens-modeling uncertainties (Bussmann et al. 2013).

To investigate the role of this relatively unexplored SMG population in the context of galaxy formation and evolution, Smolčić et al. (2015) have presented the largest sample of spectroscopically confirmed and unlensed $z>4$ SMGs (six sources) in the Cosmic Evolution Survey (COSMOS) field, where only AzTEC3 $(z=5.298)$, which has been studied in detail by Riechers et al. (2010, 2014), was excluded. To explore the dust distribution and gas kinematics, highresolution [CII] $158 \mu \mathrm{m}$ line observations have been secured toward three of these sources: J1000+0234 $(z=4.544)$, Vd-17871 $(z=4.624)$, and AzTEC/C159 $(z=4.567$, Karim et al., in prep.; hereafter K18). Based on these observations, Jones et al. (2017, hereafter J17) revealed a gas-dominated rotating disk in $\mathrm{J} 1000+0234$ and AzTEC/C159. The latter emerges as the best example of a flat rotation curve at large radius. These extreme systems, with gas-dominated rotating disks and concomitant intense star formation activity, seem to have no analog at lower redshifts; rendering their detailed study paramount to understand the formation of galaxies in the early Universe.

Here, we report the detection of ${ }^{12} \mathrm{CO}(2 \rightarrow 1)$ and ${ }^{12} \mathrm{CO}(5 \rightarrow 4)$ line emission to investigate the molecular gas properties of AzTEC/C159. ${ }^{12} \mathrm{CO}$ observations have proved to be well suited to unveil the nature of the star-forming gas of high-redshift galaxies (e.g., Frayer et al. 1998; Riechers et al. 2008; Schinnerer et al. 2008; Carilli et al. 2010; Engel et al. 2010; Riechers et al. 2011; Hodge et al. 2012, 2013; Bothwell et al. 2013; Bouché et al. 2013; Carilli \& Walter 2013; De Breuck et al. 2014; Sánchez Almeida et al. 2014; Leroy et al. 2015; Narayanan et al. 2015). Low- $J{ }^{12} \mathrm{CO}$ emission lines provide tight constraints on the mass and extent of the molecular gas reservoir, as well as on the star formation efficiency (SFE). In combination with multiple high- $J{ }^{12} \mathrm{CO}$ line detections, it is possible to explore the ${ }^{12} \mathrm{CO}$ spectral line energy distribution (SLED) and unveil the physical properties of the star-forming gas (e.g., Weiss et al. 2005, 2007; Carilli et al. 2010; Riechers et al. 2010; Papadopoulos et al. 2012b; Daddi et al. 2015). While the ${ }^{12} \mathrm{CO}(2 \rightarrow 1)$ and ${ }^{12} \mathrm{CO}(5 \rightarrow 4)$ line detections alone cannot fully constrain the shape of the ${ }^{12} \mathrm{CO}$ SLED of AzTEC/C159, they do allow us to estimate the brightness temperature ratio $r_{52}$ (e.g., Bothwell et al. 2013). This parameter can be used to obtain initial insights about the overall excitation conditions of the molecular gas in high-redshift systems (e.g., Riechers et al. 2010). Molecular line spectroscopic studies, on the other hand, can be well complemented with far-infrared (FIR) photometric information tracing the dust-obscured star-formation activity in SMGs; via FIR SED fitting, it is possible to estimate dust mass, infrared luminosity $\left(L_{\mathrm{IR}}\right)$, and hence star formation rates (SFR, e.g., Swinbank et al. 2014).

After introducing AzTEC/C159 in Sect. 2, we present the details of the observations in Sect. 3, followed by the results and discussion in Sects. 4 and 5. A summary is given in Sect. 6. Throughout, we assume a cosmology of $h_{0}=0.7, \Omega_{M}=0.3$, and $\Omega_{\Lambda}=0.7$.

\section{AzTEC/C159}

AzTEC/C159 was originally detected at the $3.7 \sigma$ level in the ASTE/AzTEC-COSMOS $1.1 \mathrm{~mm}$ survey of the inner COSMOS $1 \mathrm{deg}^{2}$ (Aretxaga et al. 2011). Long-slit spectroscopy with DEIMOS/Keck has revealed a narrow Ly $\alpha$ line, which places AzTEC/C159 at a redshift $z=4.569$ (Smolčić et al. 2015). The broadband spectral energy distribution (SED) from the optical through the FIR is consistent with a dusty star-forming galaxy, with an SFR of $\sim 700 M_{\odot} \mathrm{yr}^{-1}$, a stellar mass of $(4.5 \pm$ $0.4) \times 10^{10} M_{\odot}$, and a dust mass of $2.0_{-1.2}^{+3.0} \times 10^{9} M_{\odot}($ Smolčić et al. 2015; Gómez-Guijarro et al. 2018, hereafter GG18). High-resolution [CII] $158 \mu \mathrm{m}$ line observations $(F W H M=$ 0.36 arcsec) with the Atacama Large Millimeter/submillimeter Array (ALMA; project 2012.1.00978.S, PI: A. Karim) have revealed a double-horn profile, while the [CII] $158 \mu \mathrm{m}$ first moment and channel maps are well described by the classic pattern for rotating disks (J17, K18). AzTEC/C159 complements the sample of galaxies at $z>4$ exhibiting regular gas rotation on kiloparsec scales (i.e., disks): GN20 $(z=4.05)$, ALESS73.1 $(z=4.755), \mathrm{J} 1000+0234(z=4.542), \mathrm{Vd}-17871(z=4.622)$, and J1319+0950 $(z=6.127$, Hodge et al. 2012; De Breuck et al. 2014; Jones et al. 2017; Shao et al. 2017, K18). The kinematic signatures of rotation revealed by ALMA (J17, K18), including evidence of a flat rotation curve at large radius (J17), render 
AzTEC/C159 one of the best examples to date for an apparently rotating disk galaxy in the early Universe. We note, however, that given the achieved $\sim 2.3 \mathrm{kpc}$ resolution ALMA observations, we cannot completely rule out a merging scenario from the [C II] line dynamical analysis alone. In this context, studies that probe the physical properties of the interstellar medium (ISM), for example, molecular gas, are also key to indirectly probe the dominant mode of star formation in this system.

\section{Karl G. Jansky VLA and NOEMA Observations}

${ }^{12} \mathrm{CO}(2 \rightarrow 1)$ line observations were carried out in January 2016 with the Karl G. Jansky Very Large Array (VLA) of the NRAO in the D- and DnC-configurations (project 15B-280, PI: A. Karim). Five observing sessions of $3.5 \mathrm{~h}$ each resulted in a total of $17.5 \mathrm{~h}$, with $9.0 \mathrm{~h}$ on target. We used the quasar $3 \mathrm{C} 286$ for bandpass, delay, and flux calibration, and J1038+0512 for complex gain calibration. We made use of the $Q$-band receivers and the pair of 8-bit samplers on each VLA antenna, resulting in a pair of $1.024 \mathrm{GHz}$ bands in right and left circular polarization. These bands were overlapped by $128 \mathrm{MHz}$ to correct for the loss of signal at their edges, so that the total bandwidth was $1.92 \mathrm{GHz}$ (from 40.963 to $42.883 \mathrm{GHz}$ ), which covers the redshifted ${ }^{12} \mathrm{CO}(2 \rightarrow 1)$ line at $41.41 \mathrm{GHz}$, according to the prior redshift estimation of $z=4.567 \pm 0.002$ based on the [CII] $158 \mu \mathrm{m}$ line detection with ALMA (K18). Data were calibrated using the Common Astronomy Software Applications (CASA). Images were created with the CASA task clean and using a range of robust parameters. Ultimately, we used the image computed with robust $=1$ as it provides the best balance between spatial resolution and rms noise. This results in a data cube with a synthesized beam size of $1.70 \times 1.24$ arcsec resolution $\left(\mathrm{PA}=77.5^{\circ}\right)$ with an $\mathrm{rms}$ of $0.05 \mathrm{mJy}^{-1}$ beam ${ }^{-1}$ for $27 \mathrm{MHz}$ $\left(\sim 200 \mathrm{~km} \mathrm{~s}^{-1}\right)$ wide channel.

The ${ }^{12} \mathrm{CO}(5 \rightarrow 4)$ line was observed with the NOrthern Extended Millimeter Array (NOEMA) interferometer on December 8, 2015, and January 19, 2016, over two tracks (project 15CX001, PI: A. Karim) in D- and C-configuration. We used the WideX correlator, which covers a frequency range of $3.6 \mathrm{GHz}$. The tuning frequency of $103.51 \mathrm{GHz}$ was chosen to encompass the redshifted ${ }^{12} \mathrm{CO}(5 \rightarrow 4)$ line, considering $z=4.567 \pm 0.002$. We used the quasars 3C84 and B0906+015 as flux and phase or amplitude calibrators, respectively. The data calibration and mapping were performed with the IRAM GILDAS software packages clean and mapping. The final cube corresponds to $9.4 \mathrm{~h}$ on source out of $\sim 19 \mathrm{~h}$ of total observing time, with a synthesized beam size of $5.0 \times 2.6$ arcsec and $\mathrm{PA}=23.5^{\circ}$ (using natural weighting). We reach a sensitivity of $0.13 \mathrm{mJy} \mathrm{beam}^{-1}$ per $200 \mathrm{~km} \mathrm{~s}^{-1}$ wide channel.

\section{Analysis and results}

\subsection{CO lines}

The ${ }^{12} \mathrm{CO}(2 \rightarrow 1)$ and ${ }^{12} \mathrm{CO}(5 \rightarrow 4)$ integrated lines are detected at the 5.5 and $8.1 \sigma$ level, respectively. Figure 1 shows the intensity maps integrated over $\sim 1500 \mathrm{~km} \mathrm{~s}^{-1}$ where significant line emission was detected. The peak position of the ${ }^{12} \mathrm{CO}$ lines spatially coincides with that of the [CII] $158 \mu \mathrm{m}$ line (K18) within the positional uncertainties of $\sim 0.2$ and 0.5 arcsec, respectively. The ${ }^{12} \mathrm{CO}(2 \rightarrow 1)$ line spectrum was extracted within an ellipse of $2.1 \times 1.7 \operatorname{arcsec}(\mathrm{PA}=90 \mathrm{deg})$. This aperture contains the total extent of the [CII] $158 \mu \mathrm{m}$ line emission $(\sim 7 \mathrm{kpc}$; J17, K18) and ensures that no flux is missed. We fit Gaussians to the ${ }^{12} \mathrm{CO}$ line spectra within $\pm 850 \mathrm{~km} \mathrm{~s}^{-1}$ of the centroid (see Fig. 1) and measured an $F W H M$ of $750 \mathrm{~km} \mathrm{~s}^{-1}$ for both lines. The line emission of ${ }^{12} \mathrm{CO}(2 \rightarrow 1)$ is centered at $41.425 \pm$ $0.02 \mathrm{GHz}$, giving $z=4.565 \pm 0.003$; and the ${ }^{12} \mathrm{CO}(5 \rightarrow 4)$ centroid at $103.550 \pm 0.05 \mathrm{GHz}$ yields $z=4.565 \pm 0.003$. These values are in agreement with the redshift derived from the [CII] $158 \mu \mathrm{m}$ line of $4.567 \pm 0.002$ (K18). Finally, we measured an integrated flux of $105 \pm 19 \mathrm{mJy} \mathrm{km} \mathrm{s}^{-1}$ for the ${ }^{12} \mathrm{CO}(2 \rightarrow 1)$ line and $417 \pm 51 \mathrm{mJy} \mathrm{km} \mathrm{s}^{-1}$ for ${ }^{12} \mathrm{CO}(5 \rightarrow 4)$. We verified that the latter values differ by less than $6 \%$ from those derived by adding individual flux densities per channel within the velocity range used in the Gaussian fit.

The measured ${ }^{12} \mathrm{CO}$ integrated line flux of high-redshift galaxies is influenced by cosmic microwave background (CMB) emission. While the higher temperature of the CMB at $z=4.5$ enhances the line excitation, the background against which the ${ }^{12} \mathrm{CO}$ lines are measured also increases (e.g., da Cunha et al. 2013). For example, in a dense ISM $\left(n_{\mathrm{H}_{2}}=10^{4.2} \mathrm{~cm}^{-3}\right)$, with a gas kinetic temperature of $T_{\text {kin }}=40 \mathrm{~K}$, we can measure $70 \%$ and $80 \%$ of the intrinsic value of the ${ }^{12} \mathrm{CO}(2 \rightarrow 1)$ and ${ }^{12} \mathrm{CO}(5 \rightarrow 4)$ line, respectively. In low-density environments $\left(n_{\mathrm{H}_{2}}=10^{3.2} \mathrm{~cm}^{-3}\right)$ with $T_{\text {kin }}=18 \mathrm{~K}$, only $20 \%$ of the intrinsic value of the ${ }^{12} \mathrm{CO}(2 \rightarrow 1)$ line is detected, and less than that for ${ }^{12} \mathrm{CO}(5 \rightarrow 4)$ (da Cunha et al. 2013). Unfortunately, we lack estimates for the gas density and kinetic temperature in AzTEC/C159. It is known, however, that high SFR is likely to be associated with high temperatures of gas and dust as well as gas density (e.g., Narayanan \& Krumholz 2014). Under the assumption of thermodynamic equilibrium between the gas and dust, we would have $T_{\text {kin }} \simeq T_{\text {dust }}=37 \mathrm{~K}$ (from our mid-infrared (MIR)-millimeter SED fitting presented in Sect. 4.2). Therefore, we favor the scenario with dense gas and $T_{\text {kin }}=40 \mathrm{~K}$ that yields an increase in $S_{\mathrm{CO}(2 \rightarrow 1)}$ and $S_{\mathrm{CO}(5 \rightarrow 4)}$ by a factor [1/0.7] and [1/0.8], respectively (da Cunha et al. 2013). Consequently, we derive $S_{\mathrm{CO}(2 \rightarrow 1)}^{\text {intrinsic }}=150 \pm 27 \mathrm{mJy} \mathrm{km} \mathrm{s}^{-1}$ and $S_{\mathrm{CO}(5 \rightarrow 4)}^{\text {intrinsic }}=521 \pm 64 \mathrm{mJy} \mathrm{km} \mathrm{s}^{-1}$.

In Fig. 2 we compare the ${ }^{12} \mathrm{CO}$ and [CII] $158 \mu \mathrm{m}$ line profiles. We find that the ${ }^{12} \mathrm{CO}$ lines are as broad as the [CII] $158 \mu \mathrm{m}$ line $(\mathrm{J} 17, \mathrm{~K} 18)$, all with an $F W H M \sim 750 \mathrm{~km} \mathrm{~s}^{-1}$. There is also marginal evidence of double-horn profiles in the two ${ }^{12} \mathrm{CO}$ lines, where two peaks are close to or above the $3 \sigma$ level. To constrain the size of the ${ }^{12} \mathrm{CO}(2 \rightarrow 1)$ line emitting region, we fit a single Gaussian model to the uv data and find evidence for a point-like structure. Based on the synthesized beam size and signal-to-noise ratio $(\mathrm{S} / \mathrm{N})$ of the line detection, we used the results from Martí-Vidal et al. (2012, Eq. (7)) to estimate an upper limit of $\sim 1$ arcsec for the source size, which corresponds to an intrinsic size of $\sim 6.5 \mathrm{kpc}$ at the redshift of AzTEC/C159. An extended and possibly rotating molecular gas reservoir would be consistent with the [CII] $158 \mu \mathrm{m}$ gas extent $(\sim 7 \mathrm{kpc})$ and dynamics of AzTEC/C159 (J17, K18). However, we advise caution when interpreting our results on the ${ }^{12} \mathrm{CO}$ size and dynamics of AzTEC/C159 because of the current limitation of our ${ }^{12} \mathrm{CO}$ line observations.

\subsection{FIR properties}

Continuum emission from AzTEC/C159 was detected at $3 \mathrm{~mm}$ from our NOEMA observations. By averaging line-free channel maps, around the ${ }^{12} \mathrm{CO}(5 \rightarrow 4)$ centroid at $103.550 \mathrm{GHz}$, we derived a value of $220 \pm 40 \mu \mathrm{Jy}$. No continuum emission was detected in our VLA observations at $\sim 7 \mathrm{~mm}$; based on the noise level of the continuum image, we derive a $3 \sigma$ upper limit of $20 \mu \mathrm{Jy}$. These new millimeter data points 

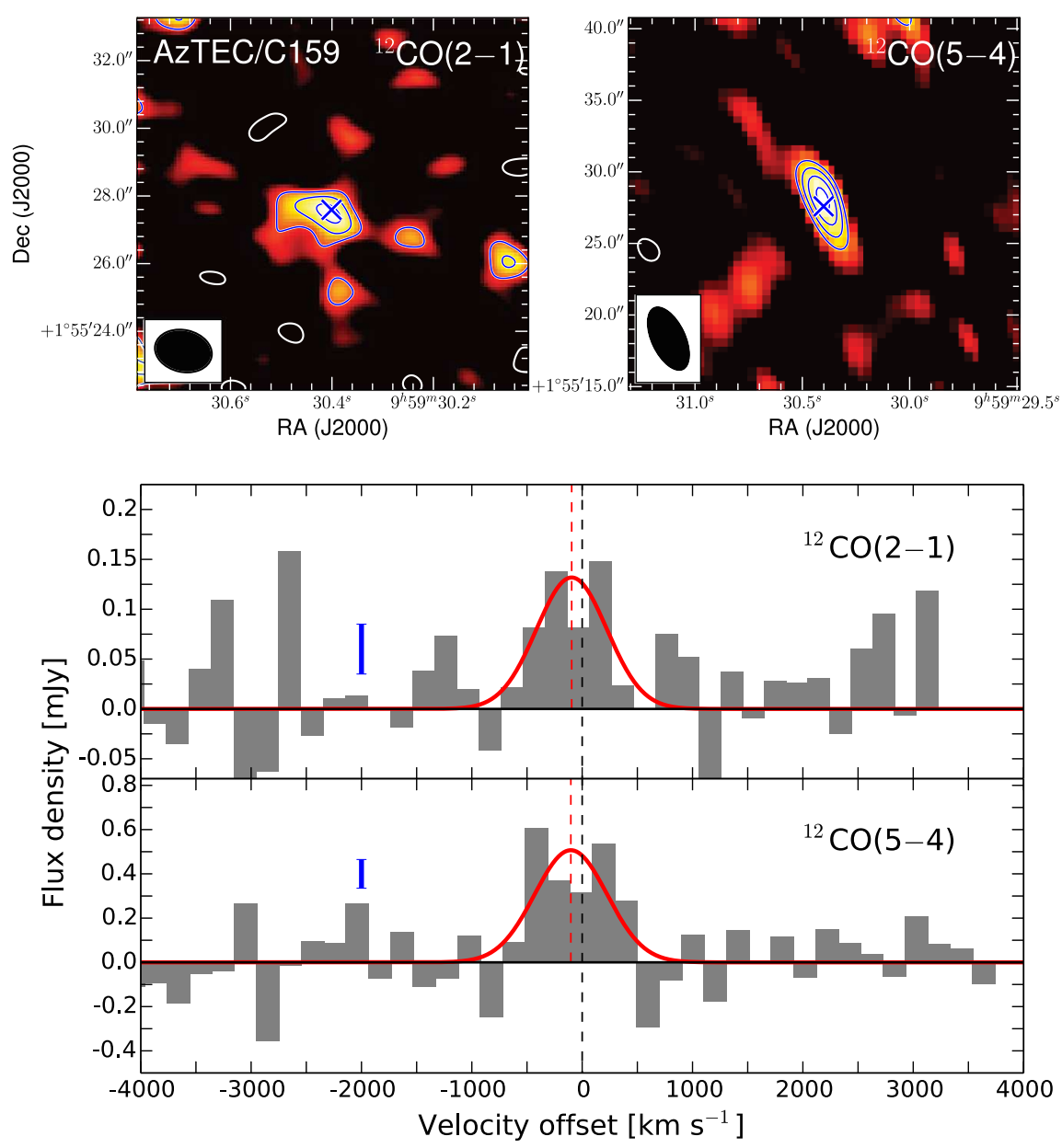

Fig. 1. Upper panels: velocity-integrated intensity maps of the ${ }^{12} \mathrm{CO}(2 \rightarrow 1)$ and ${ }^{12} \mathrm{CO}(5 \rightarrow 4)$ line. We integrated emission within the velocity range of $\pm 750 \mathrm{~km} \mathrm{~s}^{-1}$, i.e. about 8 channels, where significant line emission was detected. The contour levels of the ${ }^{12} \mathrm{CO}(2 \rightarrow 1)$ line emission map are at $[-2,2,3,4] \times 1 \sigma$, with $\sigma=0.05 \mathrm{mJy}^{-1}$ beam ${ }^{-1}$ per $200 \mathrm{~km} \mathrm{~s}^{-1}$ wide channel. Negative contours are shown in white and positive contours in blue. The beam size of $1.70 \times 1.24$ arcsec is plotted in the bottom left corner. The blue cross denotes the peak position of the [CII] $158 \mu \mathrm{m}$ emission line. In the right panel we show the velocity-integrated intensity map of the ${ }^{12} \mathrm{CO}(5 \rightarrow 4)$ line emission. Contour levels are at $[-3,3,4,5,6] \times 1 \sigma$, with $\sigma=0.13 \mathrm{mJy}_{\text {beam }}^{-1}$ per $200 \mathrm{~km} \mathrm{~s}^{-1}$ wide channel. The beam size of $5.0 \times 2.6$ arcsec is also plotted in the bottom left corner. Lower panels: ${ }^{12} \mathrm{CO}(2 \rightarrow 1)$ and ${ }^{12} \mathrm{CO}(5 \rightarrow 4)$ line spectra binned to $200 \mathrm{~km} \mathrm{~s}^{-1}$ channels. The red solid lines are Gaussian fits to the line spectra, the central position is shown by the red dashed lines. The blue error bar denotes the typical $1 \sigma \mathrm{rms}$ value.

mitigate the uncertainties in determining the FIR properties of AzTEC/C159.

We derived the infrared luminosity $\left(L_{\mathrm{IR}}\right.$; in the range $8-1000 \mu \mathrm{m})$, SFR, and dust mass $\left(M_{\text {dust }}\right)$ of AzTEC/C159 via MIR-millimeter SED fitting using the Draine \& Li (2007) dust model. We refer to Smolčić et al. (2015) for a detailed description of the fitting process. As shown in Fig. 3, in addition to our millimeter SED constraints, the $1.1 \mathrm{~mm}$ data point from the JCMT/AzTEC $1.1 \mathrm{~mm}$ survey (Scott et al. 2008), and the ALMA $870 \mu \mathrm{m}$ continuum data point (GG18), we used observations from the Herschel Space Observatory (Pilbratt et al. 2010) toward the COSMOS field: those from the Photodetector Array Camera and Spectrometer (PACS at 100 and $160 \mu \mathrm{m}$; Lutz et al. 2011) as well as from the Spectral and Photometric Imaging Receiver (SPIRE at 250, 350, and $500 \mu \mathrm{m}$; Oliver et al. 2012). We find $L_{\mathrm{IR}}=7.4_{-1.7}^{+2.1} \times 10^{12} L_{\odot}, T_{\text {dust }}=37 \pm 3 \mathrm{~K}$, $M_{\text {dust }}=2.5_{-0.5}^{+0.6} \times 10^{9} M_{\odot}$. By assuming a Chabrier initial mass function (IMF) and the relation SFR $\left[M_{\odot} \mathrm{yr}^{-1}\right]=10^{-10} L_{\mathrm{IR}}\left[L_{\odot}\right]$ (Kennicutt 1998), we estimate an SFR $=740_{-170}^{+210} M_{\odot} \mathrm{yr}^{-1}$. The derived $L_{\mathrm{IR}}$ and $T_{\text {dust }}$ are $0.3 \mathrm{dex}$ and $5 \mathrm{~K}$ higher, respectively, than the characteristic values for the full ALESS SMG sample with a median redshift of $\sim 2.5$ (Swinbank et al. 2014).
Similar to the $z>4$ SMGs presented by Smolčić et al. (2015), AzTEC/C159 lies at the high end of the $L_{\mathrm{IR}}-T_{\text {dust }}$ correlation (e.g., Chapman et al. 2005; Magnelli et al. 2012), consistent with an extrapolation of the trend for Herschel-selected $0.1<z<2.0$ infrared galaxies (Symeonidis et al. 2013).

\subsection{Molecular gas mass and star formation efficiency}

The molecular gas mass can be estimated from the relation

$M_{\mathrm{H}_{2}}=\alpha_{\mathrm{CO}} L_{\mathrm{CO}}^{\prime}$,

where $\alpha_{\mathrm{CO}}$ is the conversion factor of $\mathrm{CO}$ luminosity to $\mathrm{H}_{2}$ mass, and $L_{\mathrm{CO}}^{\prime}$ is the ${ }^{12} \mathrm{CO}(1 \rightarrow 0)$ line luminosity. The latter can be derived from our ${ }^{12} \mathrm{CO}(2 \rightarrow 1)$ line assuming that $L_{\mathrm{CO}}^{\prime}=1.18 L_{\mathrm{CO}(2 \rightarrow 1)}^{\prime}$ for typical SMG excitation conditions (consistent with the results in Sect. 4.4) and using the relation (Carilli \& Walter 2013)

$L_{\mathrm{CO}(2 \rightarrow 1)}^{\prime}=3.25 \times 10^{7} S_{\mathrm{CO}(2 \rightarrow 1)} v_{\mathrm{CO}(2 \rightarrow 1)}^{-2} D_{\mathrm{L}}^{2}(1+z)^{-1}$,

where $S_{\mathrm{CO}(2 \rightarrow 1)}$ is the integrated line flux in $\mathrm{Jy} \mathrm{km} \mathrm{s}^{-1}, v_{\mathrm{CO}(2 \rightarrow 1)}$ is the rest-frame frequency of the ${ }^{12} \mathrm{CO}(2 \rightarrow 1)$ line in $\mathrm{GHz}$, 


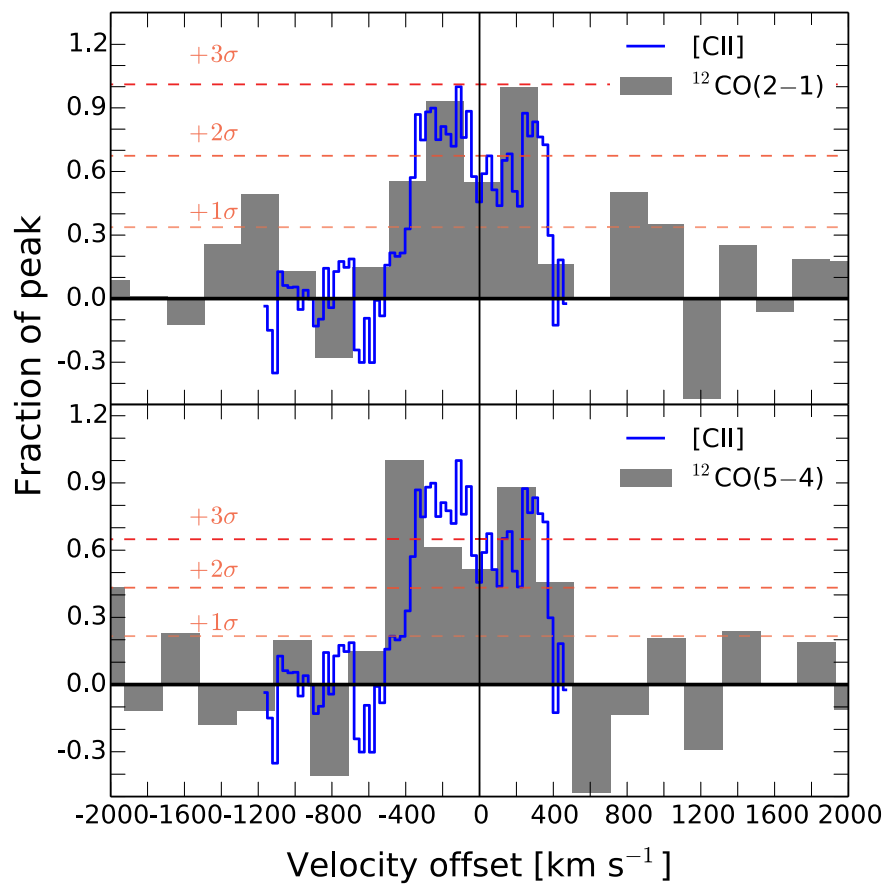

Fig. 2. [CII] $158 \mu \mathrm{m}$ line spectrum of AzTEC/C159 (blue line; J17, K18) together with the ${ }^{12} \mathrm{CO}(2 \rightarrow 1)$ and ${ }^{12} \mathrm{CO}(5 \rightarrow 4)$ detections from VLA and NOEMA, respectively. Each line is renormalized by its peak intensity. Red dashed lines show the typical noise level per channel.

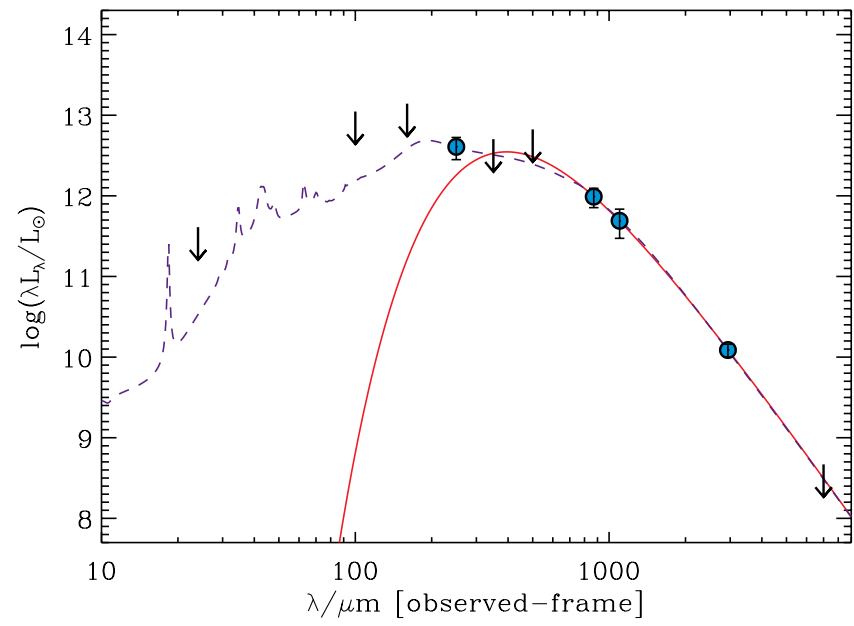

Fig. 3. Broadband SED of AzTEC/C159. The best-fit Draine \& $\mathrm{Li}$ (2007) model is shown with the blue dashed line. A modified blackbody (red line) is used to fit the FIR-to-millimeter data points. The monochromatic flux densities from Herschel PACS/SPIRE and JCMT/AzTEC observations used in the fit are listed as follows: $S_{100 \mu \mathrm{m}}<$ $6.8 \mathrm{mJy}, S_{160 \mu \mathrm{m}}<13.6 \mathrm{mJy}, S_{250 \mu \mathrm{m}}=6.2 \pm 1.9 \mathrm{mJy}, S_{350 \mu \mathrm{m}}<10.8 \mathrm{mJy}$, $S_{500 \mu \mathrm{m}}<20.4 \mathrm{mJy}$, and $S_{1.1 \mathrm{~mm}}=3.3 \pm 1.3 \mathrm{mJy}$ (Smolčić et al. 2015). We add three more submillimeter/millimeter data points from our observations: $S_{870 \mu \mathrm{m}}=6.9 \pm 0.2 \mathrm{mJy}(\mathrm{GG} 18), S_{3 \mathrm{~mm}}=220 \pm 40 \mu \mathrm{Jy}$, and $S_{7 \mathrm{~mm}}<20 \mu \mathrm{Jy}$. The downward-pointing arrows denote upper limits to the corresponding flux densities.

and $D_{\mathrm{L}}$ is the luminosity distance in Mpc. Based on $S_{\mathrm{CO}(2 \rightarrow 1)}^{\text {intrinsic }}$ (i.e., the corrected value from the $\mathrm{CMB}$ ) and Eq. (2), we derive $L_{\mathrm{CO}}^{\prime}=(3.4 \pm 0.6) \times 10^{10} \mathrm{~K} \mathrm{~km} \mathrm{~s}^{-1} \mathrm{pc}^{2}$.

The value for $\alpha_{\mathrm{CO}}$ depends on local ISM conditions and may consequently vary across different galaxy types (Daddi et al. 2010; Papadopoulos et al. 2012b; Magnelli et al. 2012; Bolatto et al. 2013). According to Papadopoulos et al. (2012b),
Table 1. Properties of AzTEC/C159.

\begin{tabular}{llcc}
\hline \hline Properties & \multirow{2}{*}{ Units } & \multicolumn{2}{c}{ Values } \\
& & ${ }^{12} \mathrm{CO}(2 \rightarrow 1)$ & ${ }^{12} \mathrm{CO}(5 \rightarrow 4)$ \\
\hline$F W H M$ & $\mathrm{~km} \mathrm{~s}^{-1}$ & $750 \pm 200$ & $770 \pm 360$ \\
Peak flux & $\mathrm{mJy}$ & $0.13 \pm 0.05$ & $0.50 \pm 0.13$ \\
Integrated flux & $\mathrm{mJy} \mathrm{km} \mathrm{s}^{-1}$ & $105 \pm 19^{a}$ & $417 \pm 51^{a}$ \\
Peak frequencies & $\mathrm{GHz}$ & $41.425 \pm 0.020$ & $103.550 \pm 0.050$ \\
\hline$z^{b}$ & $\ldots$ & $4.567 \pm 0.002$ \\
$\mathrm{RA}, \mathrm{Dec}{ }^{b}$ & $\ldots$ & $09: 59: 30.401+01: 55: 27.59$ \\
$L_{\mathrm{IR}}$ & $L_{\odot}$ & $7.4_{-1.7}^{+2.1} \times 10^{12}$ \\
$\mathrm{SFR}$ & $M_{\odot} \mathrm{yr}^{-1}$ & $740_{-170}^{+210}$ \\
$M_{\star}^{c}$ & $M_{\odot}$ & $(4.5 \pm 0.4) \times 10^{10}$ \\
$M_{\mathrm{dust}}$ & $M_{\odot}$ & $2.5_{-0.5}^{+0.6} \times 10^{9}$ \\
$M_{\mathrm{dyn}}^{d}$ & $M_{\odot}$ & $2.8_{-0.6}^{+1.1} \times 10^{11}$ \\
\hline$S_{103 \mathrm{GHz}}$ & $\mu \mathrm{Jy}$ & $220 \pm 40$ \\
$S_{41 \mathrm{GHz}}$ & $\mu \mathrm{Jy}$ & $<20$ \\
$L_{\mathrm{CO}}^{\prime}$ & $\mathrm{K} \mathrm{km} \mathrm{s}{ }^{-1} \mathrm{pc}^{2}$ & $(3.4 \pm 0.6) \times 10^{10}$ \\
$M_{\mathrm{H}{ }_{2}}\left(\alpha_{\mathrm{CO}} / 4.3\right)^{e}$ & $M_{\odot}$ & $(1.5 \pm 0.3) \times 10^{11}$ \\
$\tau_{\text {gas }} \times\left(\alpha_{\mathrm{CO}} / 4.3\right)^{e}$ & $M_{\mathrm{Nyr}}$ & $200 \pm 100$ \\
$\mu_{\mathrm{gas}} \times\left(\alpha_{\mathrm{CO}} / 4.3\right)^{e}$ & $\ldots$ & $3.3 \pm 0.7$ \\
$L_{\mathrm{IR}} / L_{\mathrm{CO}}^{\prime}$ & $L_{\odot}\left(\mathrm{K} \mathrm{km} \mathrm{s}^{-1} \mathrm{pc}^{2}\right)^{-1}$ & $216 \pm 80$ \\
\hline
\end{tabular}

Notes. ${ }^{(a)}$ After considering the CMB effect, the integrated flux density of ${ }^{12} \mathrm{CO}(2 \rightarrow 1)$ and ${ }^{12} \mathrm{CO}(5 \rightarrow 4)$ will increase by a faWector [1/0.7] and [1/0.8], respectively (see Sect. 4.1). use the corrected value, i.e., $S_{\mathrm{CO}(2 \rightarrow 1)}^{\text {intrinsic }}=150 \pm 27 \mathrm{mJy} \mathrm{km} \mathrm{s}^{-1}$ and $S_{\mathrm{CO}(5 \rightarrow 4)}^{\text {intrinsic }}=521 \pm 64 \mathrm{mJy} \mathrm{km} \mathrm{s}^{-1}$. ${ }^{(b)}$ Karim et al. (in prep.). ${ }^{(c)}$ Gómez-Guijarro et al. (2018). ${ }^{\left({ }^{(d)} J o n e s ~ e t ~ a l . ~\right.}$ (2017); within a radius of $2.9 \mathrm{kpc} .{ }^{(e)}$ Values corrected for the CMB.

the main driver of the $\alpha_{\mathrm{CO}}$ value is the average dynamical state of the molecular gas. As a result, while low values for $\alpha_{\mathrm{CO}}$ could be related to highly turbulent molecular gas, which is likely associated with merging systems, as observed in local ultra-luminous infrared galaxies (ULIRGs) with $\alpha_{\mathrm{CO}}=$ $0.8 M_{\odot} \mathrm{K}^{-1} \mathrm{~km}^{-1} \mathrm{~s} \mathrm{pc}^{-2}$ (e.g., Downes \& Solomon 1998), selfgravitating gas yields high $\alpha_{\mathrm{CO}}$ values (e.g., Papadopoulos et al. 2012a) as in star-forming spiral disks like the Milky Way with $\alpha_{\mathrm{CO}}=4.3 M_{\odot} \mathrm{K}^{-1} \mathrm{~km}^{-1} \mathrm{~s} \mathrm{pc}^{-2}$ (e.g., Abdo et al. 2010). Spatially resolved observations of nearby SFGs have also probed that $\alpha_{\mathrm{CO}}$ might vary within the galaxy itself, where central values could be significantly lower than the galaxy average (Sandstrom et al. 2013). For high-redshift unresolved sources, on the other hand, we have to rely on an average $\alpha_{\mathrm{CO}}$ that reflects the overall physical conditions of the molecular gas. Since J17 and K18 have revealed a rotationally supported gas disk in AzTEC/C159, it is likely that the bulk of the molecular gas is in self-gravitating clouds, pointing toward a Milky Way-like $\alpha_{\mathrm{CO}}$ value. We discuss the nature of $\alpha_{\mathrm{CO}}$ in this source in more detail and validate this assumption in Sect. 4.5. By adopting $\alpha_{\mathrm{CO}}=4.3 M_{\odot} \mathrm{K}^{-1} \mathrm{~km}^{-1} \mathrm{~s} \mathrm{pc}^{-2}$, we find $M_{\mathrm{H}_{2}}=(1.5 \pm$ $0.3) \times 10^{11} M_{\odot}$. Together with the stellar mass of AzTEC/C159 of $M_{\star}=(4.5 \pm 0.4) \times 10^{10} M_{\odot}(\mathrm{GG} 18)$, we estimate a gas fraction of $\mu_{\mathrm{gas}} \equiv M_{\mathrm{H}_{2}} / M_{\star}=3.3 \pm 0.7$ that is five times greater than the median for main-sequence (MS) galaxies at $z \sim 3$ (Schinnerer et al. 2016). It should be noted that even when assuming a ULIRG-like $\alpha_{\mathrm{CO}}$ prescription (and a CMB uncorrected $S_{\mathrm{CO}(2 \rightarrow 1)}$ flux), the gas fraction would be high, that is, $0.43 \pm 0.10$. The massive molecular gas reservoir of AzTEC/C159 is consistent with the general picture that large 


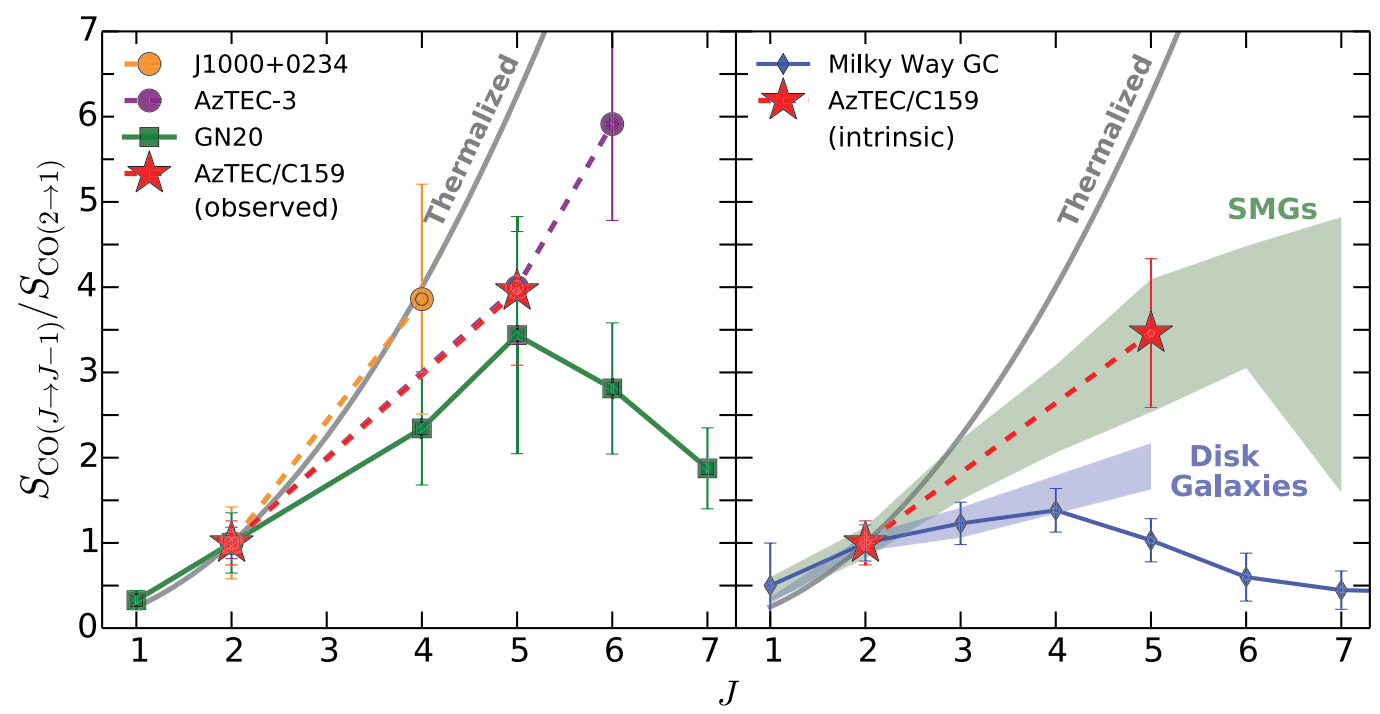

Fig. 4. Left panel: observed ${ }^{12}$ CO SLED of AzTEC/C159 and other $z>4$ SMGs for comparison: GN20 (Carilli et al. 2010), AzTEC-3 (Riechers et al. 2010), and J1000+0234 (Schinnerer et al. 2008). Gray lines show the expected trend for thermalization, i.e., LTE conditions. The dashed and solid color lines connect the data points to illustrate the shape of the different ${ }^{12} \mathrm{CO}$ SLEDs. The line fluxes have been normalized to the ${ }^{12} \mathrm{CO}(2 \rightarrow 1)$ line. Right panel: intrinsic ${ }^{12} \mathrm{CO}$ SLED of AzTEC/C159. The observed flux densities for ${ }^{12} \mathrm{CO}(2 \rightarrow 1)$ and ${ }^{12} \mathrm{CO}(5 \rightarrow 4)$ have been corrected up by $1 / 0.7$ and $1 / 0.8$, respectively, to take into account the CMB effect (see Sect. 4.1). We also present the ${ }^{12}$ CO SLED of the Milky Way galactic center (Fixsen et al. 1999), MS disk-galaxies at $z \sim 1.5$ (Daddi et al. 2015), and a sample of SMGs (median of $z=2.2$, Bothwell et al. 2013); similarly, we normalize all the line fluxes to the ${ }^{12} \mathrm{CO}(2 \rightarrow 1)$ line. Shaded regions show the dispersion of the ${ }^{12} \mathrm{CO}$ SLEDs for different SMGs and disk galaxies samples.

cool-ISM reservoirs fuel intense star formation activity at high redshift (e.g., Carilli \& Walter 2013).

We used the empirical ratio $L_{\mathrm{IR}} / L_{\mathrm{CO}}^{\prime}$ as a proxy of the SFE of AzTEC/C159, allowing a more direct comparison with the average SFE of different galaxy populations. Based on the information in Table 1 , we derive $L_{\mathrm{IR}} / L_{\mathrm{CO}}^{\prime}=(216 \pm 80)$ $L_{\odot}\left(\mathrm{K} \mathrm{km} \mathrm{s}^{-1} \mathrm{pc}^{2}\right)^{-1}$, which places it within the upper scatter of the $L_{\mathrm{IR}} / L_{\mathrm{CO}}^{\prime}$ correlation as presented by Carilli \& Walter (2013). This ratio is much higher than that observed in normal starforming disk galaxies such as nearby spirals and $z \sim(0.4-2)$ MS galaxies (between 20 and $100 L_{\odot}\left(\mathrm{K} \mathrm{km} \mathrm{s}^{-1} \mathrm{pc}^{2}\right)^{-1}$; Daddi et al. 2010; Magnelli et al. 2012), but comparable to mergerdriven starbursts such as local ULIRGs and SMGs (e.g., Genzel et al. 2010; Bothwell et al. 2013; Aravena et al. 2016, and references therein). The SFE of AzTEC/C159 is, however, similar to those of the very high-redshift starburst disk galaxies GN20 $\left(\sim 180 L_{\odot}\left(\mathrm{K} \mathrm{km} \mathrm{s}^{-1} \mathrm{pc}^{2}\right)^{-1}\right.$; Carilli et al. 2010; Hodge et al. 2012) and J1000+0234 $\left(\sim 140 L_{\odot}\left(\mathrm{K} \mathrm{km} \mathrm{s}^{-1} \mathrm{pc}^{2}\right)^{-1}\right.$; Schinnerer et al. 2008; Smolčić et al. 2015). Thus, while the extreme SFE of AzTEC/C159 seems unusual for a star-forming disk galaxy at $z<2$, at $z>4$, disk galaxies harboring vigorous SFE (such as AzTEC/C159, GN20, and J1000+0234) might be common.

\subsection{Gas excitation}

We combined our ${ }^{12} \mathrm{CO}(2 \rightarrow 1)$ and ${ }^{12} \mathrm{CO}(5 \rightarrow 4)$ line detections to obtain insights into the molecular gas excitation in AzTEC/C159. As mentioned before, at $z \sim 4.5$, the CMB emission affects the observed ${ }^{12} \mathrm{CO}$ line flux density in both lines (da Cunha et al. 2013). This could modify the ${ }^{12} \mathrm{CO}(5 \rightarrow 4) / \mathrm{CO}(2 \rightarrow 1)$ line brightness temperature ratio $\left(r_{52}\right)$ and in consequence, our interpretation. We first considered the observed line flux densities in AzTEC/C159 and compared its ${ }^{12}$ CO SLED with those of some SMGs at $z>4$, where the effect of the CMB might be considerable but no corrections have been applied yet. In Fig. 4 we plot the ${ }^{12}$ CO SLED of J1000+0234 (Schinnerer et al. 2008) and GN20 (Carilli et al. 2010), both star-forming disk galaxies with extreme SFE. We also show the ${ }^{12}$ CO SLED of AzTEC-3, a massive starburst galaxy possibly triggered by a major merger and associated with a protocluster at $z=5.3$ (Riechers et al. 2010). All these ${ }^{12}$ CO SLEDs are consistent with high gas excitation, where $r_{52}=0.63 \pm 0.16$ for AzTEC/C159.

The effect of the CMB on the ${ }^{12} \mathrm{CO}$ SLED shape below $z=2.5$ is negligible (da Cunha et al. 2013). In consequence, no corrections need to be made to the observed median ${ }^{12} \mathrm{CO}$ SLED of SMGs (median redshift of $z=2.2$ as in Bothwell et al. 2013) and disk galaxies at $z \sim 1.5$ (Daddi et al. 2010). We applied corrections to the observed flux densities of AzTEC/C159 to mitigate potential bias due to the CMB emission (see Sect. 4.1), and compared its "intrinsic" ${ }^{12}$ CO SLED with those of the aforementioned galaxy populations. As observed in Fig. 4, with $r_{52}=0.55 \pm 0.15$, the molecular gas excitation conditions of AzTEC/C159 are consistent with those observed in SMGs, and they significantly deviate from the expected trend for starforming disk-like MS galaxies at $z \sim 1.5$ (Daddi et al. 2015) and the MW (Fixsen et al. 1999). The elevated ${ }^{12}$ CO SLED of AzTEC/C159 might be a result of intrinsic processes, such as elevated gas density and kinetic temperature, which yield a higher collision rate between ${ }^{12} \mathrm{CO}$ and $\mathrm{H}_{2}$ (e.g., Narayanan \& Krumholz 2014).

\section{5. $\mathrm{CO} \rightarrow \mathrm{H}_{2}$ conversion factor}

By determining the $\alpha_{\mathrm{CO}}$ value in AzTEC/C159, we can mitigate uncertainties on our gas mass estimates and at the same time infer the overall physical conditions of its molecular gas (e.g., Papadopoulos et al. 2012a,b). Here, we constrained $\alpha_{\mathrm{CO}}$ from (a) the gas-to-dust ratio $\left(\delta_{\mathrm{GDR}}\right)$ and (b) the balance between the baryonic and dark matter content with the dynamical mass. 


\subsubsection{Gas-to-dust method}

By assuming that the molecular component dominates the ISM, that is, $M_{\mathrm{H}_{2}} \gg M_{\mathrm{HI}}+M_{\mathrm{HII}}$, the molecular gas mass can be estimated from the relation (e.g., Magnelli et al. 2012; Aravena et al. 2016)

$M_{\mathrm{gas}} \simeq M_{\mathrm{H}_{2}}=\delta_{\mathrm{GDR}} M_{\mathrm{dust}}$,

where $\delta_{\mathrm{GDR}}$ is the gas-to-dust ratio relation constrained locally by Leroy et al. (2011), which varies with metallicity as $\log \left(\delta_{\mathrm{GDR}}\right)=$ $9.4-0.85 \times[12+\log (\mathrm{O} / \mathrm{H})]$. In the absence of direct metallicity estimates for AzTEC/C159 and a robust mass-metallicity relation at such high redshift, we assumed that AzTEC/C159 has solar metallicity. Then, by combining Eqs. (1) and (3),

$\alpha_{\mathrm{CO}}=\frac{\delta_{\mathrm{GDR}} M_{\mathrm{dust}}}{L_{\mathrm{CO}}^{\prime}}$.

In Sect. 4.1 we favored a scenario with dense gas and $T_{\text {kin }}=40 \mathrm{~K}$ to correct the observed $L_{\mathrm{CO}}^{\prime}$ for the CMB effect. Here, we also explore the possibility of cool $\left(T_{\text {kin }}=14 \mathrm{~K}\right)$ and low-density molecular gas, that is, following da Cunha et al. (2013), we varied $L_{\mathrm{CO}}^{\prime}$ within the range $[3.4 \pm 0.6,12.0 \pm 2.2] \times 10^{10} \mathrm{~K} \mathrm{~km} \mathrm{~s}^{-1} \mathrm{pc}^{2}$. Based on $M_{\text {dust }}=2.5_{-0.5}^{+0.6} \times 10^{9} M_{\odot}$ (derived in Sect. 4.2) and Eq. (4), we estimate $\alpha_{\mathrm{CO}}=3.8_{-1.4}^{+2.6} M_{\odot} \mathrm{K}^{-1} \mathrm{~km}^{-1} \mathrm{~s} \mathrm{pc}^{-2}$. Note that a lower value of $\delta_{\mathrm{GDR}}$ could lower the estimated $\alpha_{\mathrm{CO}}$, but it would require unlikely supra-solar metallicity. In contrast, sub-solar metallicities would yield even higher $\alpha_{\mathrm{CO}}$.

\subsubsection{Dynamical mass method}

Another approach to constrain $\alpha_{\mathrm{CO}}$ is based on the estimation of the mass content, which should match the baryonic and dark matter content, that is, $M_{\mathrm{dyn}}=M_{\mathrm{ISM}}+M_{\star}+M_{\mathrm{DM}}$. Since we assumed that the ISM is dominated by molecular gas, $M_{\mathrm{ISM}} \simeq$ $M_{\mathrm{H} 2}$. In combination with Eq. (1), this leads to

$\alpha_{\mathrm{CO}}=\frac{M_{\mathrm{dyn}}-M_{\star}-M_{\mathrm{DM}}}{L_{\mathrm{CO}}^{\prime}}$.

The [CII] $158 \mu \mathrm{m}$ gas dynamics of AzTEC/C159 has been characterized in detail by $\mathrm{J} 17$. Via the tilted-ring fitting method, they find that at an intrinsic radius of $2.9 \mathrm{kpc} M_{\mathrm{dyn}}=\left(2.8_{-0.6}^{+1.1}\right) \times$ $10^{11} M_{\odot}$. This value agrees within the uncertainties with the value derived by $\mathrm{K} 18\left(M_{\mathrm{dyn}} \sim 2.4 \times 10^{11} M_{\odot}\right)$ using the software $3 \mathrm{D}-$ Barolo and assuming an inclination angle of $\sim 30 \mathrm{degs}$ as in J17. Within an aperture radius of $2.9 \mathrm{kpc}$, we find that $S_{\mathrm{CO}(2 \rightarrow 1)}^{\text {oberved }}=$ $91 \pm 16 \mathrm{mJy}$, which is $\sim 13 \%$ lower than that derived from the aperture that contains the full emission line (see Sect. 4.1). Consequently, as in the gas-to-dust ratio method, we consider $L_{\mathrm{CO}}^{\prime} \in[2.9 \pm 0.5,10.5 \pm 1.9] \times 10^{10} \mathrm{~K} \mathrm{~km} \mathrm{~s}^{-1} \mathrm{pc}^{2}$.

GG18 have estimated the stellar mass of AzTEC/C159 $\left([4.5 \pm 0.4] \times 10^{10} M_{\odot}\right)$ using LePHARE (Arnouts et al. 1999; Ilbert et al. 2006) and adopting the Bruzual \& Charlot (2003) stellar population synthesis models, a Chabrier (2003) IMF, and a exponentially declining star formation history ( $\mathrm{SFH}$ ). For this purpose, a substantial optical/near-IR dataset was used by GG18: the $g, r, i, z$, and $y$ bands from the Subaru Hyper Suprime-Cam imaging (HSC, Tanaka et al. 2017), near-IR $J, H$, and $K s$ bands from the UltraVISTA DR3 survey (McCracken et al. 2012), and the MIR Spitzer/IRAC 3.6 and $4.5 \mu \mathrm{m}$ bands from the Spitzer Large Area Survey with Hyper-Suprime-Cam (SPLASH, Capak et al. in prep.). As discussed in detail in GG18, this stellar mass estimate could be subject to a number of systematic uncertainties. For instance, if the stars formed in situ, it is possible that a fraction of the stellar light might be obscured by the dust and the stellar mass used here might therefore be underestimated ${ }^{1}$. GG18 have quantified the stellar mass fraction we might be missing by this effect using different prescriptions for the dust-to-stellarmass ratio (DTS). By considering the ratio derived from simulations in Popping et al. (2017) of $\log (\mathrm{DTS})=-1.8$, the stellar mass would increase by $\sim 0.4 \mathrm{dex}$; on the other hand, the median DTS ratio for local ULIRGs $\log (\mathrm{DTS})=-2.83$ (Calura et al. 2017 ) yields an increment of $\sim 1.1 \mathrm{dex}$. The original estimate for the stellar mass (combined with the SFR of $740_{-170}^{+210} M_{\odot} \mathrm{yr}^{-1}$ ) places AzTEC/C159 on the upper-end of the MS of SFGs at $z \sim 4.5$, as given by (Schreiber et al. 2015). An increase in stellar mass by $\sim 0.4$ dex would instead place AzTEC/C159 right on the MS of SFGs, while an increase by $\sim 1.1$ dex would lead to a very unlikely scenario in which AzTEC/C159 lies significantly below the MS. From this, we conclude that the stellar mass of AzTEC/C159 reported by GG18 might be underestimated by at most $\sim 0.4$ dex. By applying Eq. (5) and considering the original estimate for the stellar mass $\left([4.5 \pm 0.4] \times 10^{10} M_{\odot}\right)$ from GG18, as well as a negligible dark matter component, we derive ${ }^{2}$ $\alpha_{\mathrm{CO}}=3.9_{-1.3}^{+2.7} M_{\odot} \mathrm{K}^{-1} \mathrm{~km}^{-1} \mathrm{~s} \mathrm{pc}^{-2}$. The addition of a 0.4 dex factor in the stellar mass (i.e., $[1.0 \pm 0.4] \times 10^{11} M_{\odot}$ ) still yields a relatively high $\alpha_{\mathrm{CO}}$ value of $2.7_{-1.1}^{+2.1} M_{\odot} \mathrm{K}^{-1} \mathrm{~km}^{-1} \mathrm{~s} \mathrm{pc}^{-2}$.

Our two independent methods point toward a high value of $\alpha_{\mathrm{CO}}$. This result agrees well with our previous assumption of $\alpha_{\mathrm{CO}} \sim 4.3 \mathrm{M}_{\odot} \mathrm{K}^{-1} \mathrm{~km}^{-1} \mathrm{~s} \mathrm{pc}^{-2}$. It is known that the most important influencing factor on $\alpha_{\mathrm{CO}}$ is the average dynamical state of the molecular gas. While low values are associated with unbound gas, as observed in local ULIRGs, high values are related with self-gravitating gas (e.g., Papadopoulos et al. 2012b); hence, the molecular gas dynamics in AzTEC/C159 might be more similar to that of local star-forming disks than those of disturbed major mergers.

\section{Discussion}

Molecular line observations toward AzTEC/C159 revealed a gasrich system, elevated SFE, and high gas excitation. Its high $\alpha_{\mathrm{CO}}$ value is consistent with a self-gravitating molecular gas distribution (e.g., Papadopoulos et al. 2012a). In addition, [CII] $158 \mu \mathrm{m}$ line observations have previously revealed a gas-dominated rotating disk extending up to a radius of $\sim 3 \mathrm{kpc}$ (J17). These dynamical properties do not fit the scenario of gas-rich galaxy mergers at high redshift driving tidal torques that form dense gas regions (a condition for high gas excitation) and trigger intense star formation activity (e.g., Hopkins et al. 2006; Hayward et al. 2011, 2012). Then, the question arises which physical mechanisms might be responsible for fueling and triggering such an extreme star formation environment in this gas-rich rotating disk galaxy at $z=4.5$.

According to numerical simulations, massive rotating disk galaxies in the early Universe are a common phenomenon (e.g.,

\footnotetext{
1 Observations with high spatial resolution rest-frame UV/optical/FIR are required to map the dust and stellar light distribution and better constrain the stellar mass of AzTEC/C159. This would also allow exploring multiple interacting components (and not a massive single component) that might fit into a merger-driven star formation scenario.

2 A highly conservative error bar for $M_{\mathrm{dyn}}$ of 1 dex has been suggested by $\mathrm{J} 17$ to take into account systematic uncertainties of the method, that is, $M_{\mathrm{dyn}}=\left(2.8_{-1.9}^{+6.1}\right) \times 10^{11} M_{\odot}$. This wide range for $M_{\text {dyn }}$ also affords a wider range of inclination angles. We derive $\alpha_{\mathrm{CO}}=6.7_{-4.4}^{+6.3} M_{\odot} \mathrm{K}^{-1} \mathrm{~km}^{-1} \mathrm{spc}^{-2}$. Thus, it remains true that $\alpha_{\mathrm{CO}}$ is still higher than the prescription for ULIRGs-like systems $\left(\alpha_{\mathrm{CO}}=\right.$ $0.8 M_{\odot} \mathrm{K}^{-1} \mathrm{~km}^{-1} \mathrm{~s} \mathrm{pc}^{-2}$ ).
} 
Dekel et al. 2009b; Romano-Díaz et al. 2011). In overdense environments, the smooth infall and accretion of cold gas from cosmological filaments can build up a disk (Feng et al. 2015). This relatively smooth accretion, which dominates the mass input (Romano-Díaz et al. 2014; Anglés-Alcázar et al. 2017), can maintain an unstable dense gas-rich disk that breaks into giant clumps forming stars at a high rate (Dekel et al. 2009b). Such star formation activity might be enhanced through gravitational harassment, as advocated for the star-forming disk galaxy at $z=4.05$ GN20 (Carilli et al. 2010; Hodge et al. 2012).

The latter scenario agrees with the high gas fraction and SFE of AzTEC/C159, but it is at odds with the high gas excitation condition commonly associated with major mergers (e.g., Bournaud et al. 2015). However, as predicted by Papadopoulos et al. (2012a), high-redshift disk-like systems with extreme SFE such as AzTEC/C159 might also have high gas excitation conditions as a result of heating by turbulence and/or cosmic rays. By $z \sim 3$, the mean radiation field intensity $\langle\mathrm{U}\rangle$ in MS galaxies might become similar to that of local ULIRGs and their ${ }^{12} \mathrm{CO}$ SLEDs may look similar at this redshift (Daddi et al. 2015). We note that an elevated ${ }^{12}$ CO SLED could also be a result of AGN-driven mechanical and radiative feedback (Papadopoulos et al. 2008, 2010; Dasyra et al. 2014; Moser et al. 2016); but there is no robust evidence of an AGN in AzTEC/C159 (Smolčić et al. 2015).

It is difficult to draw a coherent conclusion on galaxy formation and evolution from this source alone. Nevertheless, AzTEC/C159 emerges as another gas-rich disk galaxy at $z \sim 4$ with high SFE and extreme ${ }^{12}$ CO SLED, together with J1000+0234 (Schinnerer et al. 2008, J17) and GN20 (Carilli et al. 2010; Hodge et al. 2012). This galaxy population complements our picture on galaxy evolution. In particular, Toft et al. (2017) suggested that these $z>4$ SMGs with cool gas reservoirs distributed in rotating disks and with high SFE, might be the progenitors of massive quenched galaxies at $z \sim 2$ that surprisingly also exhibit a rotating disk (van der Wel et al. 2011; Newman et al. 2015). To confirm this, we need to exploit the synergy between ALMA and the upcoming James Webb Space Telescope, which promise a better understanding of the multi-phase gas and stellar properties of early systems.

\section{Summary}

We reported the molecular gas properties of the star-forming disk galaxy AzTEC/C159 at $z=4.567$. Using ${ }^{12} \mathrm{CO}$ line observations of the transition levels $J=2 \rightarrow 1$ and $J=5 \rightarrow 4$ and extensive ancillary data from the COSMOS collaboration, we have found the following.

1. The molecular gas mass of AzTEC/C159 is $M_{\mathrm{H} 2}\left(\alpha_{\mathrm{CO}} / 4.3\right)=(1.5 \pm 0.3) \times 10^{11} M_{\odot}$, which yields a high gas fraction of $\mu_{\text {gas }}\left(\alpha_{\mathrm{CO}} / 4.3\right) \equiv M_{\mathrm{H}_{2}} / M_{\star}=3.3 \pm 0.7$. Its $L_{\mathrm{IR}} / L_{\mathrm{CO}}^{\prime}$ ratio of $(216 \pm 80) L_{\odot}\left(\mathrm{K} \mathrm{km} \mathrm{s}^{-1} \mathrm{pc}^{2}\right)^{-1}$, that is, $\mathrm{SFE}$, is comparable with that of local ULIRGs and SMGs (e.g., Aravena et al. 2016, and references therein).

2. The ${ }^{12} \mathrm{CO}$ lines show tentative evidence of a double-peak line profile. Their FWHM is comparable to that of the [CII] $158 \mu \mathrm{m}$ line $\left(F W H M \sim 750 \mathrm{~km} \mathrm{~s}^{-1} ; \mathrm{J} 17, \mathrm{~K} 18\right)$, which is consistent with an extended gas reservoir. However, the modest sensitivity and resolution of our observations prevent us from obtaining definitive constraints on the ${ }^{12} \mathrm{CO}$ extent and dynamics of AzTEC/C159.

3. The ${ }^{12} \mathrm{CO}(5 \rightarrow 4) / \mathrm{CO}(2 \rightarrow 1)$ line brightness temperature ratio of $r_{52}=0.55 \pm 0.15$ is consistent with the high gas excitation of the star-forming disk galaxies GN20
(Carilli et al. 2010; Hodge et al. 2012) and J1000+0234 (Schinnerer et al. 2008, J17) at $z>4$. In general, the ${ }^{12} \mathrm{CO}$ SLED of AzTEC/C159 is similar to the median for SMGs (Bothwell et al. 2013) and significantly deviates from that for star-forming disk galaxies at $z \sim 1.5$ (Daddi et al. 2015).

4. The $\mathrm{CO} \rightarrow \mathrm{H}_{2}$ conversion factor $\left(\alpha_{\mathrm{CO}}\right)$ for AzTEC/C159 is 3.9 $9_{-1.3}^{+2.7} M_{\odot} \mathrm{K}^{-1} \mathrm{~km}^{-1} \mathrm{~s} \mathrm{pc}^{-2}$ and $3.8_{-1.4}^{+2.6} M_{\odot} \mathrm{K}^{-1} \mathrm{~km}^{-1} \mathrm{~s} \mathrm{pc}^{-2}$, as given by the dynamical and gas-to-dust method, respectively. This suggests that the conditions of the ISM in AzTEC/C159 would be consistent with those of local starforming disks.

The intense star formation activity of AzTEC/C159 does not seem to be triggered by major mergers as other SMGs. Instead, its molecular gas conditions suggest that cold gas streams may fuel a gravitationally unstable gas-rich disk that harbors extreme SFE and high gas excitation. A population of high-redshift disk galaxies has been predicted by both numerical simulations and recent observational studies of quiescent rotating disk galaxies at $z \sim 2$, which are believed to be the descendants of AzTEC/C159like galaxies.

Acknowledgements. We thank Ian Smail for his detailed comments and suggestions that helped to improve the manuscript. E.F.J.A would like to thank M Krips for her support during the visit to IRAM/Grenoble and the hospitality of the DSOC in Socorro/New Mexico. Support for B.M. was provided by the DFG priority program 1573 "The physics of the interstellar medium". G.C.J. is grateful for support from NRAO through the Grote Reber Doctoral Fellowship Program. E.F.J.A, B.M., A.K., E.R.D. and F.B. acknowledge support of the Collaborative Research Center 956, subproject A1 and C4, funded by the Deutsche Forschungsgemeinschaft (DFG). C.G.G and S.T. acknowledge support from the European Research Council (ERC) Consolidator Grant funding scheme (project ConTExt, grant number: 648179). D.R. acknowledges support from the National Science Foundation under grant number AST-1614213. M.A. acknowledges partial support from FONDECYT through grant 1140099. M.J.M. acknowledges the support of the National Science Centre, Poland through the POLONEZ grant 2015/19/P/ST9/04010; this project has received funding from the European Union's Horizon 2020 research and innovation programme under the Marie Skłodowska-Curie grant agreement No. 665778. V.S. acknowledges support from the European Union's Seventh Frame-work program under grant agreement 337595 (ERC Starting Grant, "CoSMass"). The National Radio Astronomy Observatory (NRAO) is operated by Associated Universities, Inc., under cooperative agreement with the National Science Foundation. IRAM is supported by INSU/CNRS (France), MPG (Germany) and IGN (Spain).

\section{References}

Abdo, A. A., Ackermann, M., Ajello, M., et al. 2010, ApJ, 710, 133 Alaghband-Zadeh, S., Chapman, S. C., Swinbank, A. M., et al. 2012, MNRAS, 424, 2232

Anglés-Alcázar, D., Faucher-Giguère, C.-A., Kereš, D., et al. 2017, MNRAS, 470, 4698

Aravena, M., Spilker, J. S., Bethermin, M., et al. 2016, MNRAS, 457, 4406

Aretxaga, I., Wilson, G. W., Aguilar, E., et al. 2011, MNRAS, 415, 3831

Arnouts, S., Cristiani, S., Moscardini, L., et al. 1999, MNRAS, 310, 540

Banerji, M., Chapman, S. C., Smail, I., et al. 2011, MNRAS, 418, 1071

Barger, A. J., Wang, W.-H., Cowie, L. L., et al. 2012, ApJ, 761, 89

Baugh, C. M., Lacey, C. G., Frenk, C. S., et al. 2005, MNRAS, 356, 1191

Blain, A. W., Smail, I., Ivison, R. J., Kneib, J.-P., \& Frayer, D. T. 2002, Phys. Rep., 369, 111

Bolatto, A. D., Wolfire, M., \& Leroy, A. K. 2013, ARA\&A, 51, 207

Bothwell, M. S., Smail, I., Chapman, S. C., et al. 2013, MNRAS, 429, 3047

Bouché, N., Murphy, M. T., Kacprzak, G. G., et al. 2013, Science, 341, 50

Bournaud, F., Daddi, E., Weiß, A., et al. 2015, A\&A, 575, A56

Brisbin, D., Miettinen, O., Aravena, M., et al. 2017, A\&A, 608, A15

Bruzual, G., \& Charlot, S. 2003, MNRAS, 344, 1000

Bussmann, R. S. et al. 2013, ApJ, 779, 25

Capak, P., Carilli, C. L., Lee, N., et al. 2008, ApJ, 681, L53

Capak, P. L., Riechers, D., Scoville, N. Z., et al. 2011, Nature, 470, 233

Calura, F., Pozzi, F., Cresci, G., et al. 2017, MNRAS, 465, 54

Carilli, C. L., \& Walter, F. 2013, ARA\&A, 51, 105

Carilli, C. L., Daddi, E., Riechers, D., et al. 2010, ApJ, 714, 1407

Casey, C. M., Narayanan, D., \& Cooray, A. 2014, Phys. Rep., 541, 45 
Chabrier, G. 2003, PASP, 115, 763

Chapman, S. C., Blain, A. W., Smail, I., \& Ivison, R. J. 2005, ApJ, 622, 772

Coppin, K. E. K., Smail, I., Alexander, D. M., et al. 2009, MNRAS, 395, 1905

da Cunha, E., Groves, B., Walter, F., et al. 2013, ApJ, 766, 13

Daddi, E., Dannerbauer, H., Krips, M., et al. 2009a, ApJ, 695, L176

Daddi, E., Dannerbauer, H., Stern, D., et al. 2009b, ApJ, 694, 1517

Daddi, E., Bournaud, F., Walter, F., et al. 2010, ApJ, 713, 686

Daddi, E., Dannerbauer, H., Liu, D., et al. 2015, A\&A, 577, A46

Dasyra, K. M., Combes, F., Novak, G. S., et al. 2014, A\&A, 565, A46

Davé, R., Finlator, K., Oppenheimer, B. D., et al. 2010, MNRAS, 404, 1355

De Breuck, C., Williams, R. J., Swinbank, M., et al. 2014, A\&A, 565, A59

Dekel, A., Birnboim, Y., Engel, G., et al. 2009a, Nature, 457, 451

Dekel, A., Sari, R., \& Ceverino, D. 2009b, ApJ, 703, 785

Downes, D., \& Solomon, P. M. 1998, ApJ, 507, 615

Draine, B. T., \& Li, A. 2007, ApJ, 657, 810

Dwek, E., Staguhn, J. G., Arendt, R. G., et al. 2011, ApJ, 738, 36

Engel, H., Tacconi, L. J., Davies, R. I., et al. 2010, ApJ, 724, 233

Feng, Y., Matteo, T. D., Croft, R., et al. 2015, ApJ, 808, L17

Ferrara, A., Viti, S., \& Ceccarelli, C. 2016, MNRAS, 463, L112

Fixsen, D. J., Bennett, C. L., \& Mather, J. C. 1999, ApJ, 526, 207

Frayer, D. T., Ivison, R. J., Scoville, N. Z., et al. 1998, ApJ, 506, L7

Fudamoto, Y., Ivison, R. J., Oteo, I., et al. 2017, MNRAS, 472, 2028

Genzel, R., Tacconi, L. J., Gracia-Carpio, J., et al. 2010, MNRAS, 407, 2091

Gómez-Guijarro, C., Toft, S., Karim, A., et al. 2018, ApJ, 856, 121

Harrington, K. C., Yun, M. S., Cybulski, R., et al. 2016, MNRAS, 458, 4383

Harrington, K. C., Yun, M. S., Magnelli, B., et al. 2018, MNRAS, 474, 3866

Hayward, C. C., Kereš, D., Jonsson, P., et al. 2011, ApJ, 743, 159

Hayward, C. C., Jonsson, P., Kereš, D., et al. 2012, MNRAS, 424, 951

Hayward, C. C., Narayanan, D., Kereš, D., et al. 2013, MNRAS, 428, 2529

Hickox, R. C., Wardlow, J. L., Smail, I., et al. 2012, MNRAS, 421, 284

Hodge, J. A., Carilli, C. L., Walter, F., et al. 2012, ApJ, 760, 11

Hodge, J. A., Carilli, C. L., Walter, F., Daddi, E., \& Riechers, D. 2013, ApJ, 776, 22

Hopkins, P. F., Hernquist, L., Cox, T. J., et al. 2006, ApJS, 163, 1

Ikarashi, S., Ivison, R. J., Caputi, K. I., et al. 2015, ApJ, 810, 133

Ilbert, O., Arnouts, S., McCracken, H. J., et al. 2006, A\&A, 457, 841

Ivison, R. J., Lewis, A. J. R., Weiss, A., et al. 2016, ApJ, 832, 78

Jones, G. C., Carilli, C. L., Shao, Y., et al. 2017, ApJ, 850, 180

Kennicutt, J. R. C. 1998, ARA\&A, 36, 189

Kereš, D., Katz, N., Davé, R., Fardal, M., \& Weinberg, D. H. 2009a, MNRAS, 396, 2332

Kereš, D., Katz, N., Fardal, M., Davé, R., \& Weinberg, D. H. 2009b, MNRAS, 395,160

Knudsen, K. K., Kneib, J.-P., Richard, J., Petitpas, G., \& Egami, E. 2010, ApJ, 709,210

Leroy, A. K., Bolatto, A., Gordon, K., et al. 2011, ApJ, 737, 12

Leroy, A. K., Bolatto, A. D., Ostriker, E. C., et al. 2015, ApJ, 801, 25

Lutz, D., Poglitsch, A., Altieri, B., et al. 2011, A\&A, 532, A90

Magnelli, B., Saintonge, A., Lutz, D., et al. 2012, A\&A, 548, A22

Martí-Vidal, I., Pérez-Torres, M. A., \& Lobanov, A. P. 2012, A\&A, 541, A135

McCracken, H. J., Milvang-Jensen, B., Dunlop, J., et al. 2012, A\&A, 544, A156

Michałowski, M., Dunlop, J. S., Koprowski, M. P., et al. 2017, MNRAS, 469, 492

Moser, L., Krips, M., Busch, G., et al. 2016, A\&A, 587, A137

Narayanan, D., \& Krumholz, M. R. 2014, MNRAS, 442, 1411

Narayanan, D., Hayward, C. C., Cox, T. J., et al. 2010, MNRAS, 401, 1613
Narayanan, D., Turk, M., Feldmann, R., et al. 2015, Nature, 525, 496

Newman, A. B., Belli, S., \& Ellis, R. S. 2015, ApJ, 813, L7

Oliver, S. J., Bock, J., Altieri, B., et al. 2012, MNRAS, 424, 1614

Oteo, I., Ivison, R. J., Dunne, L., et al. 2016, ApJ, 827, 34

Oteo, I., Ivison, R. J., Negrello, M., et al. 2017, ApJ, submitted [arXiv: 1709.04191]

Papadopoulos, P. P., Kovacs, A., Evans, A. S., \& Barthel, P. 2008, A\&A, 491, 483

Papadopoulos, P. P., van der Werf, P., Isaak, K., \& Xilouris, E. M. 2010, ApJ, 715,775

Papadopoulos, P. P., van der Werf, P., Xilouris, E., Isaak, K. G., \& Gao, Y., 2012a, ApJ, 751, 10

Papadopoulos, P. P., van der Werf, P. P., Xilouris, E. M., et al. 2012b, MNRAS, 426, 2601

Pilbratt, G. L., Riedinger, J. R., Passvogel, T., et al. 2010, A\&A, 518, L1

Popping, G., Somerville, R. S., \& Galametz, M. 2017, MNRAS, 471, 3152

Riechers, D. A., Walter, F., Carilli, C. L., Bertoldi, F., \& Momjian, E. 2008, ApJ, 686, L9

Riechers, D. A., Capak, P. L., Carilli, C. L., et al. 2010, ApJ, 720, L131

Riechers, D. A., Hodge, J., Walter, F., Carilli, C. L., \& Bertoldi, F. 2011, ApJ, 739, L31

Riechers, D. A., Bradford, C. M., Clements, D. L., et al. 2013, Nature, 496, 329

Riechers, D. A., Carilli, C. L., Capak, P. L., et al. 2014, ApJ, 796, 84

Romano-Díaz, E., Choi, J.-H., Shlosman, I., \& Trenti, M. 2011, ApJ, 738, L19

Romano-Díaz, E., Shlosman, I., Choi, J.-H., \& Sadoun, R. 2014, ApJ, 790, L32

Sánchez Almeida, J., Elmegreen, B. G., Muñoz-Tuñón, C., \& Elmegreen, D. M. 2014, A\&ARv, 22, 71

Sandstrom, K. M., Leroy, A. K., Walter, F., et al. 2013, ApJ, 777, 5

Schinnerer, E., Carilli, C. L., Capak, P., et al. 2008, ApJ, 689, L5

Schinnerer, E., Groves, B., Sargent, M. T., et al. 2016, ApJ, 833, 112

Schreiber, C., Pannella, M., Elbaz, D., et al. 2015, A\&A, 575, A74

Scott, K. S. et al. 2008, MNRAS, 385, 2225

Shao, Y., Wang, R., Jones, G. C., et al. 2017, ApJ, 845, 138

Simpson, J. M., Swinbank, A. M., Smail, I., et al. 2014, ApJ, 788, 125s

Smolčić, V., Capak, P., Ilbert, O., et al. 2011, ApJ, 731, L27

Smolčić, V., Karim, A., Miettinen, O., et al. 2015, A\&A, 576, A127

Steinhardt, C. L., \& Speagle, J. S. 2014, ApJ, 796, 25

Strandet, M. L., Weiss, A., Vieira, J. D., et al. 2016, ApJ, 822, 80

Swinbank, A. M., Simpson, J. M., Smail, I., et al. 2014, MNRAS, 438, 1267

Symeonidis, M., Vaccari, M., Berta, S., et al. 2013, MNRAS, 431, 2317

Tacconi, L. J., Neri, R., Chapman, S. C., et al. 2006, ApJ, 640, 228

Tacconi, L. J., Genzel, R., Smail, I., et al. 2008, ApJ, 680, 246

Tanaka, M., Hasinger, G., Silverman, J. D. et al. 2017, ArXiv e-print [arXiv: 1706.00566]

Toft, S., Smolčić, V., Magnelli, B., et al. 2014, ApJ, 782, 68

Toft, S., Zabl, J., Richard, J., et al. 2017, Nature, 546, 510

van der Wel, A., Rix, H.-W., Wuyts, S., et al. 2011, ApJ, 730, 38

Walter, F., Riechers, D., Cox, P., et al. 2009, Nature, 457, 699

Walter, F., Decarli, R., Carilli, C., et al. 2012, Nature, 486, 233

Weiss, A., Walter, F., \& Scoville, N. Z. 2005, A\&A, 438, 533

Weiss, A., Downes, D., Walter, F., \& Henkel, C. 2007, in From Z-Machines to ALMA: (Sub)Millimeter Spectroscopy of Galaxies, eds. A. J. Baker, J. Glenn, A. I. Harris, J. G. Mangum, \& M. S. Yun, ASP Conf. Ser., 375, 25

Wuyts, S., Cox, T. J., Hayward, C. C., et al. 2010, ApJ, 722, 1666

Yun, M. S., Scott, K. S., Guo, Y., et al. 2012, MNRAS, 420, 957 